\title{
Chlamydia psittaci reference genes for normalisation of expression data differ depending on the culture conditions and selected time points during the chlamydial replication cycle
}

\author{
Sarah Van Lent, Daisy Vanrompay \\ Department of Animal Production, Faculty of Bioscience Engineering, \\ Ghent University, 9000 Ghent, Belgium \\ Sarah.VanLent@ugent.be
}

Received: January 22, $2016 \quad$ Accepted: November 7, 2016

\begin{abstract}
Introduction: Chlamydia psittaci is a gram-negative obligate intracellular pathogen of birds. Poultry infections lead to economic losses and can be transmitted to humans. No vaccine is available and the bacterium-host cell interaction is not completely understood. Replicating bacteria cause pneumonia, but $C$. psittaci can also be non-replicating and persistent inside the cytoplasm of avian cells. RT-qPCR provides insight into the molecular pathogenesis of both active replicating and persistent Chlamydia psittaci in birds, but requires identification of stably expressed reference genes to avoid biases. Material and Methods: We investigated the expression stability of $10 \mathrm{C}$. psittaci candidate reference genes for gene expression analysis during normal growth and penicillin-induced persistence. C. psittaci Cal10 was cultured in HeLa229 and RNA was extracted. The expression level of each candidate was examined by RT-qPCR and Cq values were analysed using geNorm. Results: The genes tyr $S$, gidA, $\operatorname{radA}$, and $16 \mathrm{~S} r R N A$ ranked among the most stably expressed. The final selected reference genes differed according to the bacterial growth status (normal growth versus persistent status), and the time points selected during the duration of the normal chlamydial developmental cycle. Conclusion: The study data show the importance of systematic validation of reference genes to confirm their stability within the strains and under the conditions selected.
\end{abstract}

Keywords: birds, Chlamydia psittaci, RT-qPCR, normalisation, references genes.

\section{Introduction}

Quantitative real-time PCR (RT-qPCR) has become a major tool to better understand the molecular pathogenesis of bacterial infections. RT-qPCR is a sensitive, efficient, and accurate technique for gene expression studies and it is an established technique for studying bacterium-host cell interactions (23). However, the accuracy and reproducibility of RTqPCR is influenced by: i) the sample amount, ii) the yield from the extraction process, iii) the RNA quality, iv) sample to sample variation, and v) reverse transcriptase efficiency (3). The expression of reference genes is affected by all sources of variation during the experimental workflow, in the same way as the expression of the genes of interest is influenced. Therefore, the use of reference genes is the preferred method to reduce the non-biological variation.
However, the normalisation step is the most problematic and ignored part of RT-qPCR. A commonly used normalisation strategy involves normalisation to a single, non-validated bacterial reference gene, such as the $16 \mathrm{~S} r R N A$ gene (13), which is generally regarded as the universal reference for data normalisation. However, evaluation of RT-qPCR candidate reference genes for expression studies in Lactobacillus casei (25), Escherichia coli (26), Bacillus cereus (18), Corynebacterium pseudotuberculosis (5), Clostridium botulinum (11), Listeria monocytogenes (21), and Staphylococcus aureus $(19,22)$ revealed that the $16 S$ rRNA gene cannot be so regarded. In fact, the expression stability of candidate reference genes should be validated specifically for each bacterial species and each experimental setting (23).

RT-qPCR analyses could help in understanding the molecular pathogenesis of Chlamydia psittaci 
infection. C. psittaci is an obligate intracellular Gramnegative bacterium that is responsible for respiratory disease in birds. $C$. psittaci infection leads to significant economic losses due to reduced feed conversion, mortality, carcass condemnation at slaughter, reduced egg production, and/or costs of antibiotic treatment (24). Currently, no vaccine is available. C. psittaci is also an important zoonotic agent infecting humans via inhalation of infected aerosols of pharyngeal or nasal excretions or dried faeces. The bacterium replicates by binary fission inside the cytoplasm of host cells but when stressed (by iron depletion, exposure to interferon gamma, and/or penicillin), the pathogen can go into a non-replicative, persistent status, and once stressors are removed, replication and bacterial excretion starts again.

Genes that are expressed at the same level at all analysed time points and conditions may be regarded as stably expressed genes. So far, for normalisation of RTqPCR data in Chlamydia such genes have only been determined for the human pathogen C. trachomatis (2). Therefore, the aim of this study was to validate reference genes for RT-qPCR studies in C. psittaci during the normal developmental cycle and during penicillin-induced persistence. Reference genes determined for normal + penicillin, i.e. genes that are stably expressed both during normal development and during penicillin-induced persistence, should be used to check whether a certain gene is up or downregulated during the persistent state compared to during normal development. This is important to further understanding of the persistent state, which could help veterinary science prevent Chlamydia spp. entering this state and thereby treat chlamydial infections more effectively.

\section{Material and Methods}

Chlamydia psittaci strain, cell culture, and infection. C. psittaci Cal10 (originally isolated from humans) was cultivated in HeLa 229 cells in $100 \mathrm{~mm}^{2}$ tissue culture dishes at $37^{\circ} \mathrm{C}$ with $5 \% \quad \mathrm{CO}_{2}$ in Dulbecco's modified Eagle's medium (DMEM, Mediatech, USA) supplemented with $10 \%$ heatinactivated foetal bovine serum (Atlanta Biologicals, USA), gentamycin (25 $\mathrm{g} \mathrm{mL}^{-1}$; Quality Biological, USA), and fungizone (1.25 $\mu \mathrm{g} \mathrm{mL}^{-1}$; Invitrogen, USA). This medium was used for normal bacterial growth conditions, while penicillin $\left(100 \mathrm{U} \mathrm{mL}^{-1}\right)$ was added to it to induce persistence. Confluent monolayers were washed with PBS, and $1 \mathrm{~mL}$ inoculum (multiplicity of infection = 1) in sucrose-phosphate-glutamic acid (SPG) (0.25 M sucrose, $10 \mathrm{mM}$ sodium phosphate, and $5 \mathrm{mM}$ L-glutamic acid) was added to one dish. SPG was added in the same $1 \mathrm{~mL}$ volume to a mock-infected dish. Both mock-infected and infected monolayers were rocked gently for $2 \mathrm{~h}$ at room temperature, washed with PBS, freshly supplemented DMEM (with or without penicillin) was added, and the cultures were incubated at $37^{\circ} \mathrm{C}$ with $5 \% \mathrm{CO}_{2}$. For all infections, addition of medium after rocking and washing marked the start time of infection.

Total RNA extraction and cDNA synthesis. Total RNA was extracted from mock-infected and infected (with or without penicillin) HeLa 229 cell cultures harvested at early ( 2 and $6 \mathrm{~h}$ p.i.), mid (12 and $18 \mathrm{~h}$ p.i.), and late $(24,32$, and $48 \mathrm{~h}$ p.i.) time points. To minimise handling time, a maximum of 15 samples were processed together. Growth medium was removed and $1 \mathrm{~mL} \mathrm{TRIzol} \mathrm{(Invitrogen)} \mathrm{was} \mathrm{added} \mathrm{to} \mathrm{each} 100 \mathrm{~mm}^{2}$ culture dish to lyse the cells. Lysates were transferred to Eppendorf tubes and $0.5 \mathrm{~mL}$ of chloroform was added to each sample. The samples were vigorously shaken for $30 \mathrm{~s}$, incubated for $5 \mathrm{~min}$ at room temperature, and centrifuged at $12000 \mathrm{~g}$ at $4^{\circ} \mathrm{C}$ for 15 min. To precipitate total RNA from the aqueous phase, $0.5 \mathrm{~mL}$ of isopropyl alcohol was added to each tube, which was subsequently shaken manually three times, incubated at room temperature for $10 \mathrm{~min}$, and centrifuged at $11500 \mathrm{~g}$ at $4^{\circ} \mathrm{C}$ for $10 \mathrm{~min}$. The pellet was washed twice with ice-cold $75 \%$ ethanol, vortexed, and centrifuged for $5 \mathrm{~min}$ at $4{ }^{\circ} \mathrm{C}$. The clean pellet was air-dried at room temperature, resuspended in $200 \mu \mathrm{L}$ RNase-free water, and quantified using a Nanodrop 2000 (Thermo Scientific, USA). All RNA samples had an $\mathrm{OD}_{260 / 280}$ between 1.8 and 2.0, indicating good RNA quality. A volume measuring $100 \mu \mathrm{L}$ of the RNA samples was treated with RNase-free DNase I (Promega, USA) following the manufacturer's protocol. Both DNase I-treated RNA samples and original RNA samples were tested by PCR (Table 1) to confirm the absence of genomic DNA in the DNasetreated RNA samples. Thereafter, cDNA was generated from $1 \mu \mathrm{g}$ of RNA with the SuperScript II RT kit (Invitrogen) with random hexamer primers (Invitrogen) following the manufacturer's protocol. RNA samples were stored at $-80^{\circ} \mathrm{C}$ and cDNA samples at $-20^{\circ} \mathrm{C}$.

Primer design and validation for RT-qPCR. Primers for the candidate reference genes were designed using Primer3 software with the following settings: amplicon size of 100-200 bp, optimal melting temperature of $60^{\circ} \mathrm{C}$, and a $\mathrm{GC}$ content of $50 \%-60 \%$ (Table 1). For each primer pair, different primer concentrations $(100 \mathrm{nM}, 150 \mathrm{nM}, 200 \mathrm{nM}, 300 \mathrm{nM}$, $400 \mathrm{nM}$, and $500 \mathrm{nM}$ ) were tested in duplicate. The concentration resulting in the best sigmoid expression curve was chosen (Table 1). Melt curve analysis was used to ensure the specificity of the primers. The RTqPCR efficiency was determined for each gene using slope analysis with a linear regression model. Standard curves were generated with serial dilutions of genomic DNA of purified EBs $\left(1 / 5=8 \mathrm{ng} \mu \mathrm{L}^{-1}, 1 / 25,1 / 125\right.$, $1 / 625,1 / 3125$, and $1 / 15625)$. The corresponding RTqPCR efficiencies (E) were calculated according to the equation $\mathrm{E}=\left(10^{(-1 / \mathrm{slope})}-1\right) \times 100(17)$. Primers selected for RT-qPCR displayed an efficiency between $90 \%$ and $110 \%$ and a coefficient of correlation greater than 0.98 . 
The Cq- values were corrected for the differences in PCR-efficiencies during the analysis.

Real-time quantitative PCR (RT-qPCR). The expression level of each candidate reference gene was examined by RT-qPCR using the Rotor-Gene Q RealTime PCR Detection System (Qiagen, Germany). Each reaction mixture contained $1 \mu \mathrm{L}$ of cDNA, the optimal primer concentration for each primer pair (Table 1), $10 \mu \mathrm{L}$ of iQ SYBR Green Supermix (Bio-Rad, USA), and $\mathrm{ddH}_{2} \mathrm{O}$ to a final volume of $20 \mu \mathrm{L}$. RT-qPCR reaction conditions were as follows: initial denaturation at $95^{\circ} \mathrm{C}$ for $3 \mathrm{~min}, 40$ cycles each consisting of $30 \mathrm{~s}$ at $95^{\circ} \mathrm{C}$ and $30 \mathrm{~s}$ at $58^{\circ} \mathrm{C}$, followed by the melting curve programme $\left(95^{\circ} \mathrm{C}\right.$ for $1 \mathrm{~min}, 55^{\circ} \mathrm{C}-95^{\circ} \mathrm{C}$ in steps of $0.5^{\circ} \mathrm{C}$ each $\left.10 \mathrm{~s}\right)$. Two biological replicates of each sample (normal infection vs. penicillin-induced persistence, each at seven different time points during the developmental cycle) were tested in duplicate. C. psittaci Cal10 genomic DNA was used as a positive control, while HeLa 229 cDNA, non-reversetranscribed C. psittaci Cal10 total RNA, and $\mathrm{ddH}_{2} \mathrm{O}$ were used as negative controls. Data analyses were carried out with geNorm software (version 2.4, Biogazelle, Belgium) on normal, penicillin, and normal + penicillin samples.
Selection of reference genes. The expression level of 10 candidate reference genes (16S rRNA, map, tyrS, hemN, hflX, gidA, gatA, fumC, $\operatorname{radA}$, and enoA) was measured for the two biological replicates of each sample. The Cq- values were used to analyse the expression stability of candidate reference genes by geNorm, implemented in the qBasePLUS software. The geNorm is based on the principle that the expression ratios of two ideal reference genes are identical in all samples tested, independent of the experimental conditions. Variation in these ratios indicates the nonstable expression of one or both reference genes. Therefore, geNorm determines the level of pairwise variation for each candidate reference gene with all other candidate reference genes (M-value). Genes with a low M-value are the most stably expressed. Sequential removal of the least stable gene generates a ranking of the candidate reference genes according to their stable expression. The geNorm also calculates the pairwise variation $V_{n / n+1}$ to determine the ideal number of reference genes for normalisation (23). The value 0.15 was set as the cut-off below which the inclusion of an additional control gene was considered not to result in a significant improvement of the normalisation.

Table 1. Primers used for RT-qPCR analysis

\begin{tabular}{|c|c|c|c|c|c|c|c|}
\hline Gene & Primer & Primer sequence $\left(5^{\prime}-3^{\prime}\right)$ & $\begin{array}{l}\text { Amplicon } \\
\text { size (bp) }\end{array}$ & $\begin{array}{c}\mathrm{Tm} \\
\left({ }^{\circ} \mathrm{C}\right)\end{array}$ & $\begin{array}{l}\mathrm{GC} \\
(\%)\end{array}$ & $\begin{array}{c}\text { Primer } \\
\text { concentration }(\mathrm{nM})\end{array}$ & $\begin{array}{c}\text { Efficiency } \\
(\%)\end{array}$ \\
\hline \multirow[t]{2}{*}{$16 S r R N A$} & 16SrRNA-1 & TGTACAAGGCCCGGGAACGTA & 156 & 59.9 & 57.1 & 200 & 95.4 \\
\hline & 16SrRNA-2 & GGCCAGTACAGAAGGTAGCA & & 58.0 & 55.0 & 200 & \\
\hline \multirow[t]{2}{*}{ enoA } & enoA-F & AGCCGCAACTTTAGGACGA & 187 & 60.9 & 52.6 & 500 & 90.1 \\
\hline & enoA-R & ATCAGCACCCATACGCACAG & & 62.1 & 55.0 & 500 & \\
\hline \multirow[t]{2}{*}{ gatA } & gatA-F & GCGTTAGGTTCCGATACAG & 165 & 55.9 & 52.6 & 200 & 94.9 \\
\hline & gatA-R & GGCGACATCTTCAACAAC & & 54.9 & 50.0 & 200 & \\
\hline \multirow[t]{2}{*}{ hemN } & hemN-F & TTTACACATGCGGCCTGAC & 170 & 60.7 & 52.6 & 500 & 101.2 \\
\hline & hemN-R & CAATGGCTTGGTAACCTGCT & & 60.1 & 50.0 & 500 & \\
\hline \multirow[t]{2}{*}{ tyrS } & tyrS-F & TGGGACAGGCTTATGGTTTG & 169 & 60.9 & 50.0 & 200 & 96.6 \\
\hline & tyrS-R & CGTGCGACTTTAGGCACTTC & & 61.0 & 55.0 & 200 & \\
\hline \multirow[t]{2}{*}{ fumC } & fumC-F & CTTGCATACCGCCAGAGAGT & 170 & 60.4 & 55.0 & 200 & 103.9 \\
\hline & fumC-R & CAACCCAACGCAATGTGA & & 60.1 & 50.0 & 200 & \\
\hline \multirow[t]{2}{*}{ gidA } & gidA-F & GATCTCCGGGTTGTTCTTCA & 100 & 60.1 & 50.0 & 400 & 97.9 \\
\hline & gidA-R & GAACGTGGTTTCCCAATCAG & & 60.4 & 50.0 & 400 & \\
\hline \multirow[t]{2}{*}{$h f l X$} & hflX-F & CGTAAGGCTAAAGAG & 181 & 57.3 & 55.0 & 500 & 97.8 \\
\hline & hflX-R & TTGCCCACTAGGAAG & & 57.2 & 55.0 & 500 & \\
\hline \multirow[t]{2}{*}{$\operatorname{radA}$} & $\operatorname{radA}-\mathrm{F}$ & GTCGCCGCCTAATAGGGTAA & 108 & 61.3 & 55.0 & 500 & 105.6 \\
\hline & radA-R & ACCATAGAGCTGCGAGAGGA & & 60.1 & 55.0 & 500 & \\
\hline \multirow[t]{2}{*}{ map } & map-F & AAACGCGTCTGTCAAGCATC & 156 & 61.4 & 50.0 & 200 & 92.2 \\
\hline & map-R & ACCCACACCGTGACCTACAA & & 61.3 & 55.0 & 200 & \\
\hline
\end{tabular}

Tm- melting temperature

Table 2. Candidate reference genes

\begin{tabular}{lll}
\hline Gene symbol & Function & Pathways \\
\hline map & Mitogen-activated protein kinase & Protein phosphorylation \\
\hline tyrS & Tyrosine-tRNA ligase & Catalysation of the attachment of an amino acid to its cognate tRNA molecule \\
\hline $16 S r R N A$ & 16S ribosomal RNA subunit & Translation \\
\hline hemN & Coproporphyrinogen III oxidase & Coproporphyrinogen III decarboxyation \\
\hline r $f X$ & GTPase & May have a role during protein synthesis or ribosome biogenesis \\
\hline enoA & DNA recombination/repair protein & DNA repair, homologous recombination \\
\hline fumC & Component of enolase & Glycolysis \\
\hline gatA & Fumarase C & Citric acid cycle \\
\hline
\end{tabular}




\section{Results}

Choice and transcript profiles of candidate reference genes. Reference genes for $C$. psittaci were validated during normal and penicillin-induced persistence conditions, at early ( 2 and $6 \mathrm{~h}$ p.i.), mid (12 and $18 \mathrm{~h}$ p.i.), and late (24, 32 and $48 \mathrm{~h}$ p.i.) time points. The candidate reference genes were chosen based on the reference genes tested for C. trachomatis and the housekeeping genes used for the multi locus sequence typing of Chlamydiales $(2,16)$. To minimise the risk of co-regulation, 10 candidate genes were selected by the following criteria: widely spread on the chromosome, involved in different pathways, and not adjacent to putative outer membrane, secreted, or hypothetical proteins that might be under diversifying selection (Table 2). The gene encoding the $16 \mathrm{~S}$ rRNA was the most abundantly expressed (only 3.97 cycles to reach the cycle threshold), while eno $A$ and fumC were the least abundant transcripts (31.83 and 31.2 cycles respectively) (Fig. 1); the lower the Cq-value was, the higher was the transcript level, and the higher the Cqvalue was, the lower was the transcript level; thus 3.97 was a high abundant transcript level and 31.83 was a low abundant transcript level. Both $\operatorname{radA}$ and $\operatorname{tyr} S$ transcript levels showed the lowest, whereas eno $A$ and fumC transcript levels the highest variation in Cqvalues $(10.67,10.59,15.99$, and 19.84 cycles respectively). The wide range of transcript levels of the candidate reference genes confirmed that no single candidate reference gene was constantly expressed at the different conditions and time points analysed. This implicated the need for using multiple reference genes.

Stability of reference genes expression. The stability of the transcript levels of the candidate reference genes was determined using geNorm. The programme calculated the average expression stability value (M-value) for each candidate reference gene during normal, penicillin, and normal + penicillin conditions (Fig. 1). For each condition, the stability value for each candidate reference gene was determined for early, mid, late, and all time points. In addition to the M-value, geNorm calculated also a $V_{n / n+1}$-value to determine the optimal number of reference genes for accurate normalisation for each condition (Fig. 2). A $\mathrm{V}_{\mathrm{n} / \mathrm{n}+1}$ lower than 0.15 indicates that an additional reference gene $\left(\mathrm{V}_{\mathrm{n}+1}\right)$ has no significant effect (1). If the pairwise variation was higher than 0.15 , than geNorm advised the use of the number of reference genes with the lowest $V_{n / n+1}$ value. The lowest and highest $\mathrm{M}$-value of the reference genes for all the samples (normal + penicillin) and for normal and penicillin conditions separately, as well as which reference genes to use for each condition, are listed in Table 3. gatA was excluded from the analysis, as it was not transcribed in all samples. In general, gidA had the lowest M-value (highest stability) for six conditions, while eno $A$ had the highest M-value (lowest stability) for 6 conditions. Genes tyrS, gidA, $\operatorname{rad} A$, and $16 S \mathrm{rRNA}$ were among the reference genes suggested to be used in $11,9,8$, and 8 out of the 12 tested conditions respectively, while genes map, $h f l X$, enoA, hem $N$, and fum $C$ were suggested to be used in $5,4,2,1$, and none of the tested conditions. enoA and hem $N$ were unique reference genes for the normal condition. No gene was unique for the penicillin-induced persistence condition, although in general the reference genes proposed for use during the normal condition at a specific time point differed from the ones proposed for the penicillininduced persistent condition at the corresponding time point. Including more different samples resulted in less stably expressed reference genes. For example, the lowest M-value of the normal + penicillin condition was higher than that of normal and penicillin separately for early, mid, late, and all time points. In addition, the lowest M-value of normal, penicillin, and normal + penicillin was the highest for all time points together compared to early, mid, and late time points separately. Late and all time point samples were less stable than early and mid time point samples, as late and all time points showed the highest M-values. Consequently, the more conditions were analysed, the more reference genes were needed to normalise the data accurately (two to five reference genes for normal and penicillin conditions separately, while four to five reference genes for normal + penicillin conditions together).

Table 3. Overview of reference genes for three conditions: normal, penicillin, and normal + penicillin

\begin{tabular}{|c|c|c|c|c|c|}
\hline Condition & Time point & Lowest M-value & Highest M-value & Reference genes & $\mathrm{V}_{\mathrm{n} / \mathrm{n}+1}<0.15 ?$ \\
\hline \multirow{4}{*}{ Normal } & early & $0.432($ hem $N)$ & $2.126(h f l X)$ & enoA, tyrS, hemN & No \\
\hline & mid & $0.398(16 S r R N A)$ & $2.204(\mathrm{map})$ & gidA, tyrS, hflX, 16S rRNA & No \\
\hline & late & $0.718($ gidA $)$ & $2.832($ enoA) & $16 S \mathrm{rRNA}$, map, tyrS, radA, gidA & No \\
\hline & all & $1.024($ tyr $S)$ & $3.012($ enoA $)$ & $h f l X, \operatorname{rad} A$, gidA, tyrS & No \\
\hline \multirow{4}{*}{ Penicillin } & early & $0.488($ gidA $)$ & $2.243($ hemN) & tyrS, map, gidA & Yes \\
\hline & mid & $0.151($ gidA $)$ & $1.169($ fumC) & $\operatorname{rad} A, \operatorname{gid} A$ & Yes \\
\hline & late & $0.631($ tyrS $)$ & $2.657($ enoA $)$ & $\operatorname{radA}, h f l X, 16 S \mathrm{rRNA}$, tyrS & No \\
\hline & all & 0.997 (tyrS) & $2.719($ enoA $)$ & map, $16 S$ rRNA, radA, tyrS & No \\
\hline \multirow{4}{*}{ Normal + penicillin } & early & $0.1817($ gidA $)$ & 2.263 (fumC) & eno $A, 16 S$ rRNA, map, tyrS, gidA & No \\
\hline & mid & $0.502($ gidA $)$ & $1.822($ fumC) & $16 S \operatorname{rRNA}$, tyrS, $\operatorname{rad} A$, gidA & No \\
\hline & late & $1.081(\operatorname{gid} A)$ & $2.745($ eno $A)$ & hflX, $16 S$ rRNA, tyrS, $\operatorname{rad} A$, gidA & No \\
\hline & all & $1.226($ tyrS $)$ & $2.846($ eno $A)$ & $16 S \operatorname{rRNA}$, map, radA, gidA, tyrS & No \\
\hline
\end{tabular}


Early

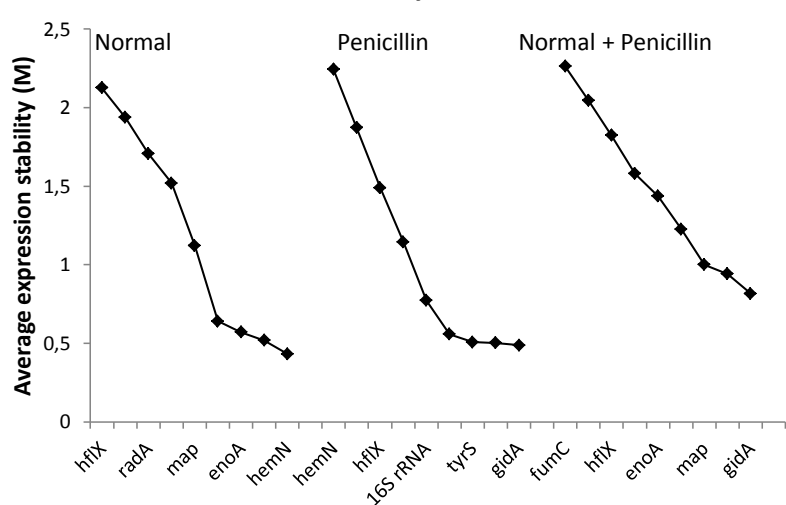

Late

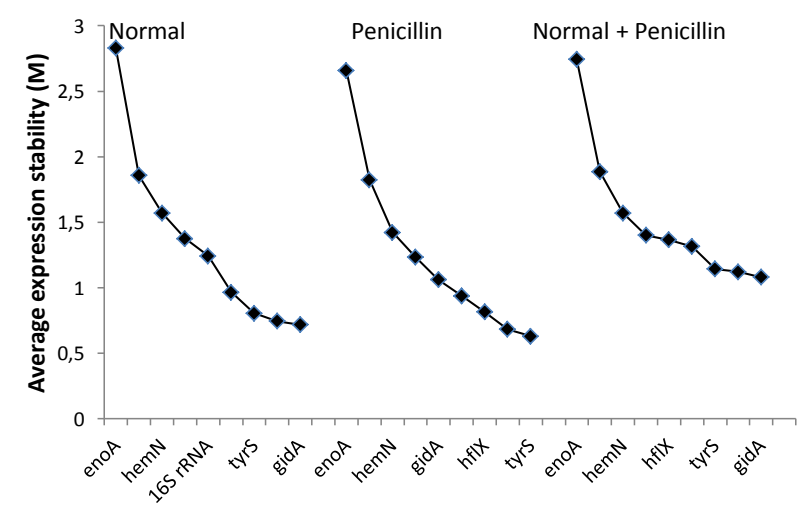

Mid

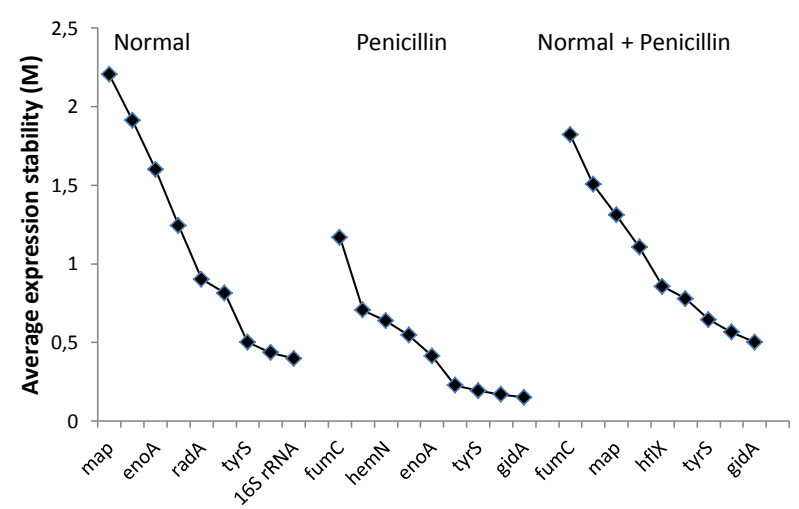

Early, mid and late

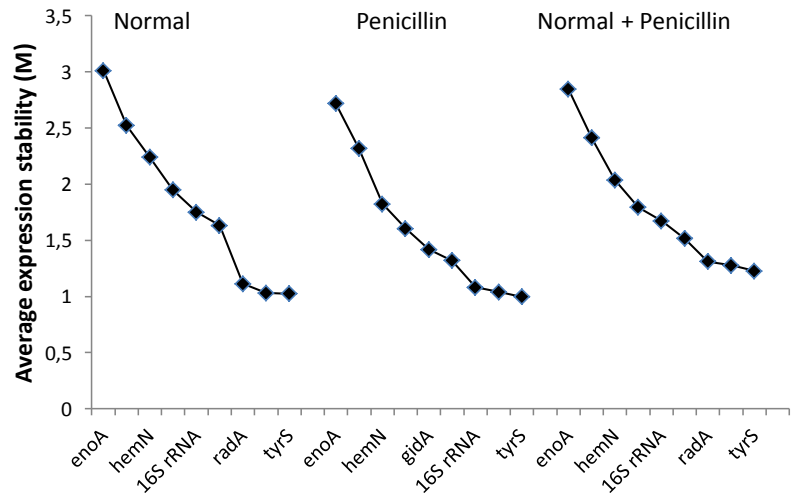

Fig. 1. Stability ranking of the reference genes during normal, penicillin and normal + penicillin conditions by geNorm. Genes are ranked from left to right in order of increasing expression stability (decreasing M-value)
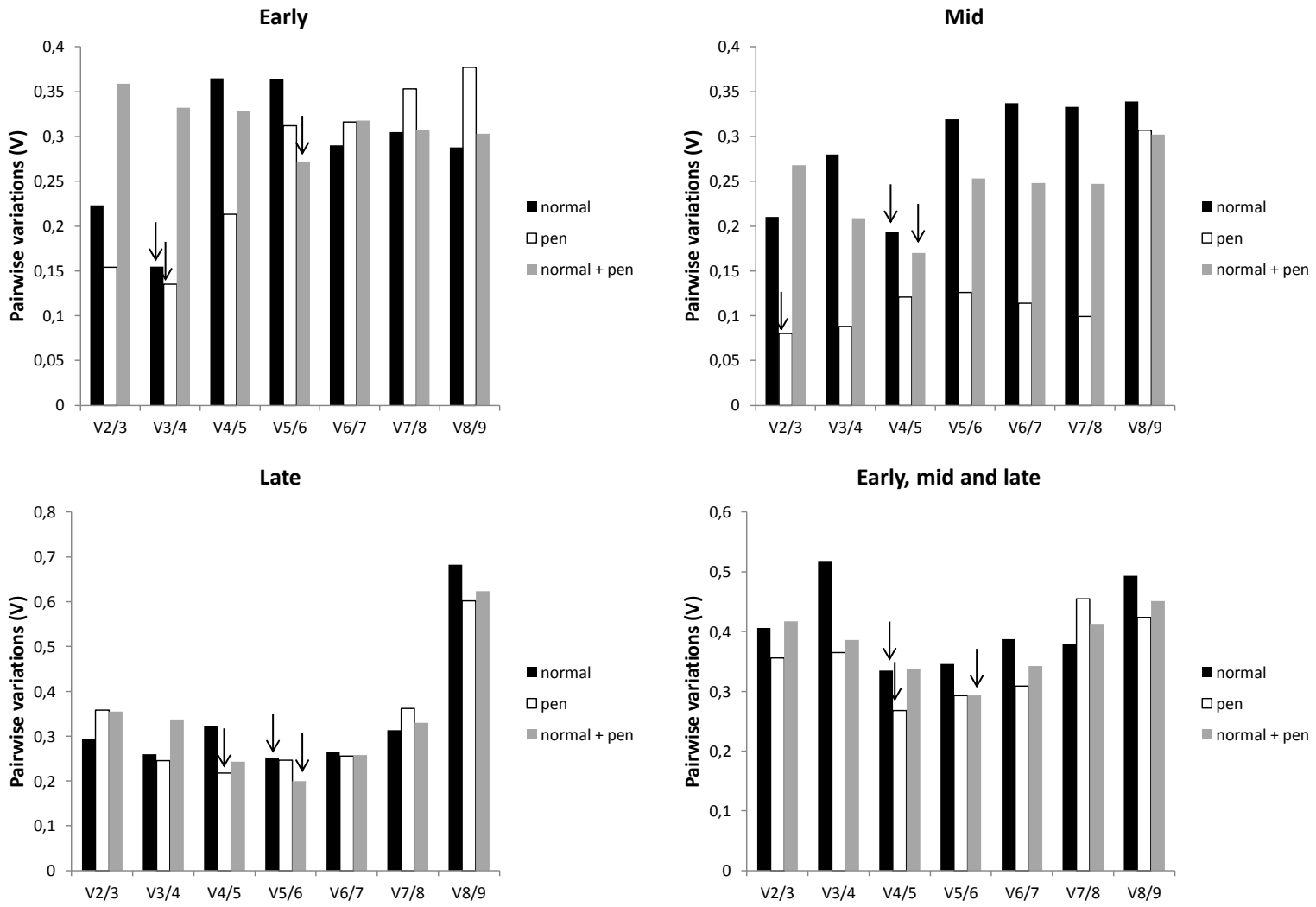

Fig 2. Determination of the optimal number of reference genes required for reliable normalisation by geNorm

The pairwise variation $\left(\mathrm{V}_{\mathrm{n} / \mathrm{n}+1}\right)$ was calculated stepwise between normalisation factors based on the $\mathrm{n}$ and $(\mathrm{n}+1)$ most stable expressed reference genes. According to the geNorm developers, a variation of $<0.15$ indicates no significant contribution of an additional control gene to the normalisation factor. If $\mathrm{V}_{\mathrm{n} / \mathrm{n}+1}$ is higher than 0.15 , then the lowest $\mathrm{V}_{\mathrm{n} / \mathrm{n}+1}$ is the optimal number. The optimal number of control genes is illustrated by arrows. 


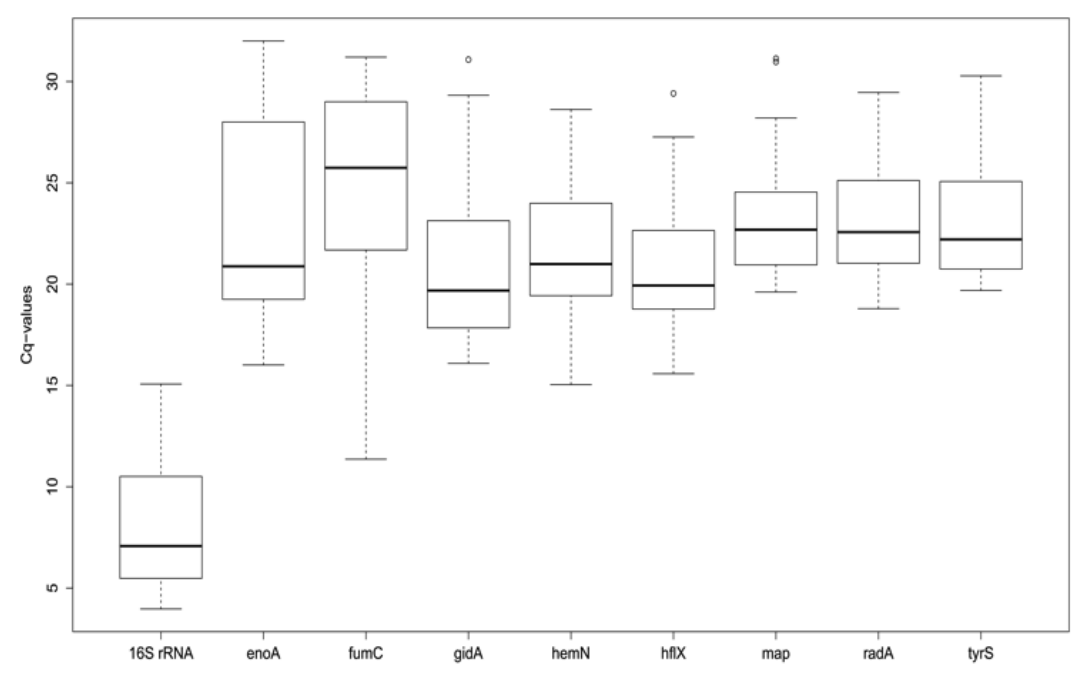

Fig. 3. Transcript levels of candidate reference genes

\section{Discussion}

A RT-qPCR is an accurate and sensitive tool for studying gene expression in bacterial pathogens (4). Unfortunately, normalisation is still the most persistent problem for real-time quantification. Different normalisation methods are available. The use of genomic DNA is less suited to normalising experimental variations, as the gDNA is determined on DNA samples. Consequently, it cannot be used to correct for differences in RNA extraction and RT-PCR efficiencies (13). Therefore, the use of reference genes is advised (23). However, it is essential to validate reference genes for each bacterial species and each specific experiment to be able to control for nonbiological variation and thereby obtain accurate and reliable gene expression data. Selection of unstable, unvalidated reference genes can result in miscalculations of gene expression levels and lead to incorrect conclusions (6). Several publications have underlined the importance of using multiple reference genes, as no single, universal reference gene exists. Nevertheless, in many recently published papers mRNA levels were still normalised by a single reference gene $(4,8,9,12)$, mostly $16 S r R N A$. In fact, none of the previously mentioned studies which used $16 S$ rRNA as a reference gene showed data on the stability of the gene in the experimental setting utilised. Other reports justified the choice of the $16 S$ rRNA as reference gene only by referring to another study, usually performed under different experimental conditions with other strains.

Borges et al. (2), validated reference genes for performing gene expression analyses in C. trachomatis, but reference genes have not been validated for gene expression studies in other Chlamydia species. Therefore, we investigated the suitability of 10 candidate reference genes for future gene expression analysis in C. psittaci Cal10. The expression of the $16 \mathrm{~S}$ $r R N A$ gene, extensively used as reference gene in Chlamydia spp. gene expression studies $(1,7,8,10,14$, 15, 20), and of nine other C. psittaci genes (map, tyrS, hem $N, h f l X, \operatorname{gid} A, \operatorname{gat} A$, fumC, $\operatorname{rad} A$, and enoA) was studied during both normal bacterial growth conditions and penicillin-induced persistence.

Our data confirm the finding that the best-suited reference genes differ among experimental conditions, as the most stably expressed reference gene (lowest Mvalue) varied for each experimental group. The most striking observation was that the tyrS gene was suggested as a reference gene for all conditions but one, namely not for the mid time point during penicillininduced persistence. We have no reasonable explanation for the latter observation. 16S rRNA was suggested as reference gene in only 8 out of the 12 tested conditions. This result is in alignment with an earlier study, in which it was found that $16 S \mathrm{rRNA}$ was the most stable reference gene for $C$. trachomatis under normal conditions, but its expression was highly unstable during stress conditions (2). In addition, the reference genes to be used for $C$. psittaci gene expression analyses differ from those described for C. trachomatis gene expression analysis (2). As also demonstrated by Vandesompele et al. (23), measuring expression levels by using multiple reference genes was more accurate than just using one. The effect of potential regulations of single genes is decreased by the use of multiple reference genes, and improves the reproducibility of relative gene expression analysis.

In conclusion, we successfully identified reference genes, which can be used for C. psittaci gene expression analysis during the normal developmental cycle and during penicillin-induced persistence. The importance of proper reference gene evaluation for RT- 
qPCR data normalisation is emphasised by our data and therefore we strongly advise carrying out systematic validation of reference genes to confirm their stability within the strains and under the conditions selected.

Conflict of Interests Statement: The authors declare that there is no conflict of interests regarding the publication of this article.

Financial Disclosure Statement: Sarah Van Lent was supported by Research Foundation Flanders (FWO) (11E8213N).

Animal Rights Statement: None required.

\section{References}

1. Belland R.J., Nelson D.E., Virok D., Crane D.D., Hogan D., Sturdevant D., Beatty W.L. Harland D.C.: Transcriptome analysis of chlamydial growth during IFN-gamma-mediated persistence and reactivation. Proc Natl Acad Sci 2003, 100, 15971-1596.

2. Borges V., Ferreira R., Nunes A., Nogueira P., Borrego M.J., Gomes J.P.: Normalization strategies for real-time expression data in Chlamydia trachomatis. J Microbiol Methods 2010, 82, 256-264.

3. Bustin S.A.: Quantification of mRNA using real-time reverse transcription PCR ( RT-PCR ): trends and problems. J Mol Endocrinol, 2001, 29, 23-39.

4. Bustin S.A.: Quantitative real-time RT-PCR - a perspective. J Mol Endocrinol 2005, 34, 597-601.

5. Carvalho D.M., de Sá P.H., Castro T.L.P., Pinto A., Gil D. J. P., Bagano P., Bastos B., Costa L. F. M., Meyer R., Silva A., Azevedo V., Ramos R. T. J., Pacheco L. G. C.: Reference genes for RT-qPCR studies in Corynebacterium pseudotuberculosis identified through analysis of RNA-seq data. Antonie Van Leeuwenhoek 2014, 106, 605-614.

6. Dheda K., Huggett J.F., Chang J.S., Kim L.U., Bustin S.A., Johnson M.A., Rook G.A.W., Zumla A.: The implications of using an inappropriate reference gene for real-time reverse transcription PCR data normalization. Anal Biochem 2005, 344, 141-143.

7. Douglas A.L., Hatch T.P..: Expression of the transcripts of the sigma factors and putative sigma factor regulators of Chlamydia trachomatis L2. Gene 2000, 247, 209-214.

8. Ferreira R., Borges V., Nunes A., Borrego M.J., Gomes J.P.: Assessment of the load and transcriptional dynamics of Chlamydia trachomatis plasmid according to strains' tissue tropism. Microbiol Res 2013, 168, 333-339.

9. Filipe Almeida F., Borges V., Ferreira R., Borrego M.J., Gomes J.P., Mota L.J.: Polymorphisms in inc proteins and differential expression of inc genes among Chlamydia trachomatis strains correlate with invasiveness and tropism of lymphogranuloma venereum isolates. J Bacteriol 2012, 194, 6574-6585.

10. Goellner S., Schubert E., Liebler-Tenorio E., Hotzel H., Saluz H.P., Sachse K.: Transcriptional response patterns of Chlamydophila psittaci in different in vitro models of persistent infection. Infect Immun 2006, 74, 4801-4808

11. Kirk D.G., Palonen E., Korkeala H., Lindström M.: Evaluation of normalization reference genes for RT-qPCR analysis of spo0A and four sporulation sigma factor genes in Clostridium botulinum Group I strain ATCC 3502. Anaerobe 2014, 26, 14-19.

12. Kiselev A.O., Skinner M.C., Lampe M.F.: Analysis of pmpD expression and PmpD post-translational processing during the life cycle of Chlamydia trachomatis serovars A, D, and L2. PLoS One 2009, 4, e5191.

13. Mannonen L., Markkula E., Puolakkainen M.: Analysis of Chlamydia pneumoniae infection in mononuclear cells by reverse transcription-PCR targeted to chlamydial gene transcripts. Med Microbiol Immunol 2011, 200, 143-54.

14. Mathews S., George C., Flegg C., Stenzel D., Timms P.: Differential expression of ompA, ompB, pyk, nlpD and Cpn0585 genes between normal and interferon-gamma treated cultures of Chlamydia pneumoniae. Microb Pathog 2001, 30, 337-345.

15. Nicholson T.L., Chiu K., Stephens R.S.: Chlamydia trachomatis lacks an adaptive response to changes in carbon source availability. Society 2004, 72, 4286-4289.

16. Pannekoek Y., Morelli G., Kusecek B., Morré S.A., Ossewaarde J.M., Langerak A.A., van der Ende A.: Multi locus sequence typing of Chlamydiales: clonal groupings within the obligate intracellular bacteria Chlamydia trachomatis. BMC Microbiol 2008, 8, 42.

17. Pfaffl M.W.: A new mathematical model for relative quantification in real-time RT-PCR. Nucleic Acids Res $2001,29, \mathrm{e} 45$.

18. Reiter L., Kolstø A.B., Piehler A.P.: Reference genes for quantitative, reverse-transcription PCR in Bacillus cereus group strains throughout the bacterial life cycle. J Microbiol Methods $2011,86,210-217$

19. Sihto H.M., Tasara T., Stephan R., Johler S.: Validation of reference genes for normalization of qPCR mRNA expression levels in Staphylococcus aureus exposed to osmotic and lactic acid stress conditions encountered during food production and preservation. FEMS Microbiol Lett 2014, 356, 134-140.

20. Suchland R.J., Jeffrey B.M., Xia M., Bhatia A., Chu H.G., Rockey D.D., Stamm W.E.: Identification of concomitant infection with Chlamydia trachomatis IncA-negative mutant and wild-type strains by genomic, transcriptional, and biological characterizations. Infect Immun 2008, 76, 5438-5446.

21. Tasara T., Stephan R.: Evaluation of housekeeping genes in Listeria monocytogenes as potential internal control references for normalizing mRNA expression levels in stress adaptation models using real-time PCR. FEMS Microbiol Lett 2007, 269, 265-272.

22. Valihrach L., Demnerova K.: Impact of normalization method on experimental outcome using RT-qPCR in Staphylococcus aureus. J Microbiol Methods 2012, 90, 214-216.

23. Vandesompele J., De Preter K., Pattyn F., Poppe B., Van Roy N., De Paepe A., Speleman F.: Accurate normalization of real-time quantitative RT-PCR data by geometric averaging of multiple internal control genes. Genome Biol 2002, 3, research0034.1.

24. Vanrompay D., Butaye P., Van Nerom A., Ducatelle R., F. Haesebrouck: The prevalence of Chlamydia psittaci infections in Belgian commercial turkey poults. Vet Microbiol 1997, 54, $85-93$.

25. Zhao W., Li Y., Gao P., Sun Z., Sun T., Zhang H.: Validation of reference genes for real-time quantitative PCR studies in gene expression levels of Lactobacillus casei Zhang. J Ind Microbiol Biotechnol 2011,38, 1279-1286.

26. Zhou K., Zhou L., Lim Q.E., Zou R., Stephanopoulos G., Too H.-P.: Novel reference genes for quantifying transcriptional responses of Escherichia coli to protein overexpression by quantitative PCR. BMC Mol Biol 2011, 12, 18. 